\title{
MAGE3 and Survivin activated dendritic cell immunotherapy for the treatment of non-small cell lung cancer
}

\author{
DONG $\mathrm{LI}^{1}$ and SONG HE $\mathrm{HE}^{2,3}$ \\ ${ }^{1}$ Department of Cardiothoracic Surgery, Central Hospital of Zibo, Zibo, Shandong 250012;
${ }^{2}$ Maanshan Center for Clinical Laboratory; ${ }^{3}$ Maanshan Municipal Hospital Group, Maanshan, Anhui 243000, P.R. China
}

Received June 17, 2015; Accepted October 24, 2016

DOI: $10.3892 / \mathrm{ol} .2018 .8362$

\begin{abstract}
Dendritic cell (DC) immunotherapy is an optimal cancer treatment, resulting in its emergence as a therapeutic choice; however, there are limited studies investigating dual antigen-pulsed DC immunotherapy in non-small cell lung cancer (NSCLC). In order to determine the effect of a recombinant melanoma-associated antigen (rMAGE-3) and recombinant Survivin (rSurvivin) peptide-pulsed DC immunotherapy in patients with NSCLC, the present clinical study was performed. DC immunotherapy was generated from the monocytes of patients with NSCLC and primed with rMAGE-3 and rSurvivin peptides. The present open-label, non-randomised study enrolled 16 patients with histologically confirmed stage I-IIIB NSCLC between December 2013 and October 2014. A prime immunotherapy $\left(9.1 \times 10^{7}\right.$ cells/dose $)$ and a single boost $\left(8.2 \times 10^{7}\right.$ cells/dose $)$ were administered 1 month apart intradermally and the patients were evaluated for immunological and clinical response. DC immunotherapy was well tolerated, with no serious adverse events. There was a single incidence of grade 1 fever, chills and fatigue. Out of the 16 patients enrolled, 11 patients showed stable disease and 5 showed disease progression. There was a significant increase in IFN- $\gamma$ expression on day 60 vs. day $0(\mathrm{P}=0.048)$. An increasing trend in the mean cluster of differentiation (CD)4:CD8 values of day 30 and day 90 was observed, but this was not significant. The present study established that DCs primed with rMAGE-3 and rSurvivin may be used in NSCLC treatment. However, a larger study is required to address prominent issues, including secretion of immunosuppressive cytokines and mechanisms of tumour escape from immune surveillance. Several factors associated with the manufacturing and quality of immunotherapy also require standardisation.
\end{abstract}

Correspondence to: Dr Dong Li, Department of Cardiothoracic Surgery, Central Hospital of Zibo, 54 West of Gongqingtuan Road, Zhangdian, Zibo, Shandong 250012, P.R. China

E-mail: dongli3301@gmail.com

Key words: cancer immunotherapy, cancer vaccine, dendritic cells, non-small cell lung cancer

\section{Introduction}

In total, $80-85 \%$ of all patients with lung cancer are diagnosed with non-small cell lung cancer (NSCLC), resulting in NSCLC being the most common type of lung cancer (1). In addition, NSCLC has remained a crucial cause of mortality in patients with carcinoma, despite notable advances in early diagnosis and treatment modalities. In the light of the discovery of dendritic cells and current approaches in two major areas, tumour-associated antigens (TAAs) and adoptive cellular targeting, immunotherapy has resulted in an improved prognosis for the treatment of patients with NSCLC at an advanced stage of disease (2). Among the numerous immunotherapies, dendritic cell (DC)-based adoptive immunotherapy has emerged as the most viable option for such patients. DCs are professional antigen-presenting cells that efficiently activate $\mathrm{T}$ lymphocytes by presenting the antigens to immature cluster of differentiation (CD) $4^{+}$cells via major histocompatibility complex (MHC) class II and $\mathrm{CD}^{+}$cells through MHC class I $(3,4)$. Studies have reported that DCs may be generated from autologous monocytes $\left(\mathrm{CD} 14^{+}\right.$cells condensed by apheresis) by making use of a culture medium that is complimented with interleukin (IL) 4, granulocyte-macrophage colony-stimulating factor (GM-CSF) or IL-13 (1-4) and pulsed ex vivo with TAAs to elicit a potent $\mathrm{T}$ cell-mediated immune response and protect against additional tumour challenges (5-8).

However, collective data on the use of dendritic cell-based immunotherapy for the treatment of NSCLC are limited, and to the best of our knowledge, none of the previously reported clinical trials have exclusively evaluated DC immunotherapies in NSCLC $(9,10)$. Studies have suggested that Survivin and MAGE-3 are overexpressed in NSCLC and may play a vital role in tumourigenesis $(11,12)$. Therefore, the present study was performed to identify the immunological response along with the efficacy and harmlessness of the restorative vaccination using autologous DCs pulsed with recombinant melanoma-associated antigen (rMAGE-3) and recombinant Survivin (rSurvivin) peptide in patients with NSCLC.

\section{Materials and methods}

Sample study and design. A total of 16 NSCLC patients were enrolled in the present open-label non-randomised study. All patients had histologically-confirmed diagnoses of stage I-IIIB 
disease. Patients that had stable disease at the time of screening and had completed definitive therapy (surgical, medical or multimodal) were eligible to participate in the present study. The Ethics Committee of the Central Hospital of Zibo (Zibo, China) approved the study protocol; thus, prior to the start of the current study, written informed consent was collected from all participating patients. The present study followed all the required modifications under the International Conference on Harmonisation and Good Clinical Practice guidelines and was in agreement with the Declaration of Helsinki, 1975. Between December 2013 and October 2014, patients with disease duration of 6 weeks to 3 years (average, 8 months) after definitive therapy were enrolled in the present study. A heterogeneous group of patients was selected with respect to medical history, stage of disease, risk of recurrence and treatment of primary disease. Characteristics of the patients are summarised in Table I.

Measurable immunological response to DC immunotherapy was the primary endpoint, and obtaining comparative immunological data from different NSCLC patients that had received a definitive therapy was the secondary endpoint. To evaluate the inhibitory effects of persistent tumour load and to assess the impact of previous radiotherapy and chemotherapy on immunological responses, the patients were primarily stratified according the therapy they had received. Heterogeneity of the patients and small sample size can prevent meaningful evaluation of therapeutic effects. Therefore, it was important to incorporate the immunotherapy into the therapeutic plan of the patient, with little time commitment and risk. Routine safety laboratory measurements were performed to evaluate clinical tolerability, and adverse events were assessed according to the National Cancer Institute Cancer Therapy Evaluation Program and Common Terminology Criteria for Adverse Events (13).

Dosing schedule. The protocol followed, including the dose used and the route and interval of administration, was selected on the basis of the methods used previously (14). The target dose selected was $10^{8}$ DCs pulsed with rMAGE3 + rSurvivin in a total $3 \mathrm{ml}$ volume, and a prime immunotherapy followed by one boost immunotherapy were intradermally administered in the thigh 1 month apart. Overall, 16 prime and boost injections containing $9.1 \times 10^{7} \mathrm{DCs}$ and $8.2 \times 10^{7} \mathrm{DCs}$, respectively, were administered. Following immunisation, patients were monitored for $2 \mathrm{~h}$ in the outpatient clinic for immediate unexpected adverse events.

Preparation of monocyte-derived DCs (MODCs). The DC immunotherapies were developed as described by Hirschowitz et al (14). Briefly, each patient was subjected to a 3 -h leukapheresis procedure and $1-3 \times 10^{10}$ peripheral blood mononuclear cells (PBMCs) were drawn. The cells were then placed in a tissue culture flask at a density of $1 \times 10^{6}$ cells $/ \mathrm{cm}^{2}$ in the presence of $1 \%$ human serum albumin (Baxter Healthcare, Deerfield, IL, USA). Subsequent to incubating the cells in $5 \% \mathrm{CO}_{2}$ at $37^{\circ} \mathrm{C}$ for $2 \mathrm{~h}$, the flask was washed with sterile phosphate-buffered saline (PBS) to isolate non-adherent cells. Adherent cells were then resuspended in a clinical grade CellGro DC medium (CellGenix, Breisgau, Germany) containing 1,000 U/ml GM-CSF (CellGenix), $50 \mathrm{ng} / \mathrm{ml}$ IL-4 (CellGenix) and were incubated for 5 days in $5 \% \mathrm{CO}_{2}$ at $37^{\circ} \mathrm{C}$. On the fifth day, DCs were split into 2 aliquots, one for rMAGE3 and the other for rSurvivin. TAA peptides at a concentration of $10 \mu \mathrm{g} / \mathrm{ml}$ in $10 \mathrm{ml}$ PBS were individually added to every aliquot and then incubated at $37^{\circ} \mathrm{C}$ for $2 \mathrm{~h}$. The aliquots were then transferred to a single vial. To induce DC maturation, cytokine cocktail, IL-1 $\beta$ (Peprotech, Rocky Hill, NJ, USA), IL-6 (Peprotech), tumour necrosis factor- $\alpha$ (TNF- $\alpha$; Peprotech), interferon- $\gamma$ (IFN- $\gamma$; LG Life Sciences, Gurgaon, Haryana, India), prostaglandin E2 (PGE2; Sigma Aldrich; Merck Millipore, Darmstadt, Germany) and poly I:C (Sigma Aldrich; Merck Millipore) were added to the culture between days 5 and 7. DCs were later bathed twice and then resuspended in $1 \mathrm{ml}$ PBS. Identification of the morphology and immunophenotyping for CD14, CD83, CD86, CD1a and human leukocyte antigen-antigen D related (HLA-DR) was performed in MODCs and later detected for its sterility. The final formulation contained rMAGE3-primed and rSurvivin-primed DCs in the ratio of $1: 1$, and the total cell concentration was $5 \times 10^{6} \mathrm{DCs}$ in each dose. All 5 doses were prepared at a time and were frozen using automated cryopreservation.

Generation of recombinant proteins. Procreation of the cDNAs encoding MAGE-3 or Survivin into the pCTP vector was performed as previously described (15). It was observed that Escherichia coli BL21 (DE3) had both the antigens (MAGE-3 and Survivin) that were in the structure of $6 \mathrm{x}$-His-attached fusion proteins. Nickel-nitrilotriacetic acid column chromatography (Qiagen, Hilden, Germany) was used to purify the antigens. Endotoxin $<1.0 \mathrm{EU} / \mu \mathrm{g}$ in limulus amoebocyte lysate test (catalog no. 88282; Thermo Fischer Scientific, Waltham, MA, USA), according to the manufacturer's instructions, and $>95 \%$ purity in SDS-PAGE analysis were performed for the quality control authentication of all antigens. Working solutions were prepared by dissolving $0.6 \mathrm{mg}$ of each peptide in $30 \mathrm{ml}$ dimethyl sulfoxide (Wak-chemie Medical GmbH, Steinbach, Germany) and $270 \mathrm{ml}$ sterile water, resulting in a final concentration of $2 \mathrm{mg} / \mathrm{ml}$.

In vitro characterisation. Each DC immunotherapy was subjected to sterility testing and characterisation for the expression of CD14, CD86, CD205 and HLA-DR. Mycoplasma contamination was checked with the use of a MycoAlert Mycoplasma Detection kit (Lonza, Auckland, New Zealand). A kinetic chromogenic limulus amoebocyte lysate test (Lonza) was used in order to identify the endotoxin, according to the manufacturer's instructions.

Evaluation of the phenotypes of DCs. The phenotypes of mature DCs, immature DCs and monocytes were determined using one- or two-colour fluorescence analysis. In total, $3 \times 10^{5}$ cells were resuspended in $50 \mu \mathrm{l}$ of buffer containing PBS, $2 \%$ foetal calf serum (FCS) and $1 \%$ sodium azide. The cells were then incubated with $10 \mu \mathrm{l}$ of appropriate phycoerythrin-labelled monoclonal antibodies (mAbs) at a dilution of 1:100 or fluorescein isothiocyanate (FITC) (HLA-DR: catalog no. 130-098-176; clone, AC122; CD14: catalog no. 130-110-576; clone, REA599; CD86: catalog no. 130-098-182; clone, FM95; CD205: catalog no. 130-104-772; clone, HD30; Miltenyi Biotec, Singapore) at $4^{\circ} \mathrm{C}$ for $30 \mathrm{~min}$. Subsequent to incubation, the cells were washed twice and resuspended in $500 \mu \mathrm{l}$ of assay buffer. The 
Table I. Patient characteristics.

\begin{tabular}{|c|c|c|c|c|c|}
\hline $\begin{array}{l}\text { Patient } \\
\text { code }\end{array}$ & Age, years & Histology & TNM stage & Prior treatment received & $\begin{array}{c}\text { Duration from } \\
\text { last treatment to DC } \\
\text { immunotherapy, months }\end{array}$ \\
\hline $1 \mathrm{XU}$ & 53 & Squamous & IIIA & Chemotherapy + radiation therapy & 8 \\
\hline 2LI & 63 & Squamous & IIIA & Chemotherapy + radiation therapy & 5 \\
\hline $3 \mathrm{XB}$ & 50 & Adenocarcinoma & IIIB & Neo/surgery/adjuvant chemotherapy & 31 \\
\hline $4 Y U$ & 65 & Bronchoalveolar & IB & Surgery & 6 \\
\hline $5 \mathrm{NC}$ & 72 & Adenocarcinoma & IB & Surgery & 8 \\
\hline 6QL & 59 & Adenocarcinoma & IA & Neo/surgery/adjuvant chemotherapy & 5 \\
\hline $7 \mathrm{ZY}$ & 66 & Adenocarcinoma & IIIA & Surgery/adjuvant chemotherapy & 4 \\
\hline $8 X L$ & 57 & Squamous & IIIA & Chemotherapy + radiation therapy & 4 \\
\hline 9YP & 52 & Adenocarcinoma & IIB & Chemotherapy + radiation therapy & 12 \\
\hline $10 \mathrm{LO}$ & 65 & Adenocarcinoma & IB & Chemotherapy + radiation therapy & 5 \\
\hline $11 \mathrm{XE}$ & 61 & Squamous & IA & Chemotherapy + radiation therapy & 7 \\
\hline $12 \mathrm{NZ}$ & 58 & Adenocarcinoma & IIIA & Neo/surgery/adjuvant chemotherapy & 3 \\
\hline $13 \mathrm{HE}$ & 70 & Squamous & IIIA & Surgery/adjuvant chemotherapy & 3 \\
\hline $14 \mathrm{PX}$ & 56 & Squamous & IIIA & Neo/surgery/adjuvant chemotherapy & 5 \\
\hline $15 \mathrm{SZ}$ & 71 & Adenocarcinoma & IIIA & Chemotherapy + radiation therapy & 7 \\
\hline $16 Y U$ & 62 & Adenocarcinoma & IIB & Surgery/chemotherapy & 11 \\
\hline
\end{tabular}

TNM, tumour-node-metastasis; DC, dendritic cell.

fluorescence was analysed by a flow cytometer (FACSCalibur; BD Biosciences, Franklin Lakes, NJ, USA). There was a build up of 15,000 events for every sample, in addition to delineation of the number of positive cells. DCs were characterised using human HLA-DR-, CD14-, CD86- and CD205-specific mAbs (Miltenyi Biotec) and control immunoglobulins G1 and G2a (IgG1 and IgG2a; BD Biosciences).

Intracytoplasmic IFN- $\gamma$ detection assay. The procedure used was determined by Kern et al (16) for the intracellular staining of IFN- $\gamma$ released by lymphocytes. Briefly, $5 \times 10^{6} \mathrm{CD} 14$-peripheral mononuclear cells were obtained prior to the first injection (T0) and subsequent to the fourth injection (T4). Co-culturing of the cells was then performed for $18 \mathrm{~h}$ with $1 \times 10^{6}$ mature MODCs pulsed with rMAGE3 + rSurvivin. Protein secretion was blocked during the last $3 \mathrm{~h}$ using $10 \mu \mathrm{mol}$ of monensin (Sigma Aldrich; Merck Millipore). T0 and T4 cells that were not exposed to rMAGE3 + rSurvivin were used as controls. Intermingling of ionomycin $(500 \mathrm{ng} / \mathrm{ml}$; Sigma Aldrich; Merck Millipore) and phorbol myristate acetate (PMA; $50 \mathrm{ng} / \mathrm{ml}$; Sigma Aldrich; Merck Millipore) was performed with the cell suspensions in a correspondent experimental lay down. Subsequent to harvesting, washing and permeabilising the cells with a permeabilisation agent (Immunotech Laboratories, Inc.), according to the manufacturer's protocol, the cells were double-stained with IFN- $\gamma$-specific or CD69-specific antibody labelled with phycoerythrin (catalog no. 130-098-901; clone, FN50; Miltenyi Biotec) and CD3-specific antibody (catalog no., 130-098-162; clone, BW264/56; dilution, 1:15; Miltenyi Biotec) labelled with FITC. IgG1 antibodies were utilized as isotype controls. The samples were examined using a flow cytometer (FACSCalibur; BD Biosciences).
Ratio of $\mathrm{CD}^{+}$and $\mathrm{CD} 8^{+}$cells. The CD4 and CD8 lymphocyte count was analysed in accordance with the technique described by Bapsy et al (17). Briefly, 2-3 $\mathrm{ml}$ of peripheral blood was incubated with anti-human CD3-PC5, CD4-FITC, CD8-PE and CD16-FITC mAbs (BD Biosciences). Subsequent to staining the cells, they were fixed with $1 \%$ paraformaldehyde and examination was performed using FACSCalibur flow cytometer and CellQuest Pro software (BD Biosciences). Lymphocytes are characterised by their side and forward light scattering properties; therefore, the analysis and acquisition gates were limited to the lymphocyte gate. Cells that expressed CD markers were acquired and analysed in the FL1 or FL2 logarithmic scale by using the set gates.

Delayed-type hypersensitivity test. rMAGE3 + rSurvivin-pulsed DCs and unpulsed DCs were administered intra-dermally into the forearm, at the time of T0 and T4. Erythema $>1.5 \mathrm{~cm}$ and skin induration $48 \mathrm{~h}$ after intradermal injection were considered as positive delayed-type hypersensitivity.

Response evaluation. Patients were followed-up by the primary physicians. The follow-up included physical examination and routine history. Chest X-rays or computed tomography (CT) scans were also obtained for assessment depending on the signs and symptoms of tumour recurrence or at regular intervals. Toxicity was graded according to World Health Organisation criteria.

Statistical evaluation. Paired Student's $t$-test was used to examine the data. $\mathrm{P}<0.05$ was considered to indicate a statistically significant difference. The correlation between two 
Table II. Treatment characteristics and DC vaccine-associated clinical events.

\begin{tabular}{|c|c|c|c|c|c|}
\hline $\begin{array}{l}\text { Patient } \\
\text { code }\end{array}$ & Recurrence & $\begin{array}{c}\text { Time to recurrence } \\
\text { from treatment, } \\
\text { months }\end{array}$ & $\begin{array}{c}\text { Time to recurrence } \\
\text { from DC immunotherapy, } \\
\text { months }\end{array}$ & $\begin{array}{l}\text { Survival from } \\
\text { treatment, } \\
\text { months }\end{array}$ & $\begin{array}{c}\text { Survival from } \\
\text { DC immunotherapy, } \\
\text { months }\end{array}$ \\
\hline $1 \mathrm{XU}$ & No & 22 & - & - & NA \\
\hline $2 \mathrm{LI}$ & No & 12 & - & - & NA \\
\hline $3 \mathrm{XB}$ & Yes & - & 7 & 12 & 7 \\
\hline $4 Y U$ & No & 17 & - & - & NA \\
\hline $5 \mathrm{NC}$ & No & 25 & - & 15 & 6 \\
\hline 6QL & No & 5 & 3 & 16 & 12 \\
\hline $7 Z Y$ & Yes & 15 & 3 & 12 & NA \\
\hline 8XL & No & 20 & - & - & NA \\
\hline $9 Y P$ & Yes & 14 & - & - & NA \\
\hline $10 \mathrm{LO}$ & No & 17 & - & - & NA \\
\hline $11 \mathrm{XE}$ & No & 13 & - & - & NA \\
\hline $12 \mathrm{NZ}$ & No & 25 & - & - & NA \\
\hline $13 \mathrm{HE}$ & No & 16 & - & - & NA \\
\hline $14 \mathrm{PX}$ & No & - & - & - & NA \\
\hline $15 \mathrm{SZ}$ & Yes & 11 & 16 & NA & NA \\
\hline $16 Y U$ & Yes & - & 8 & 11 & NA \\
\hline
\end{tabular}

NA, not applicable; -, not observed; DC, dendritic cell.
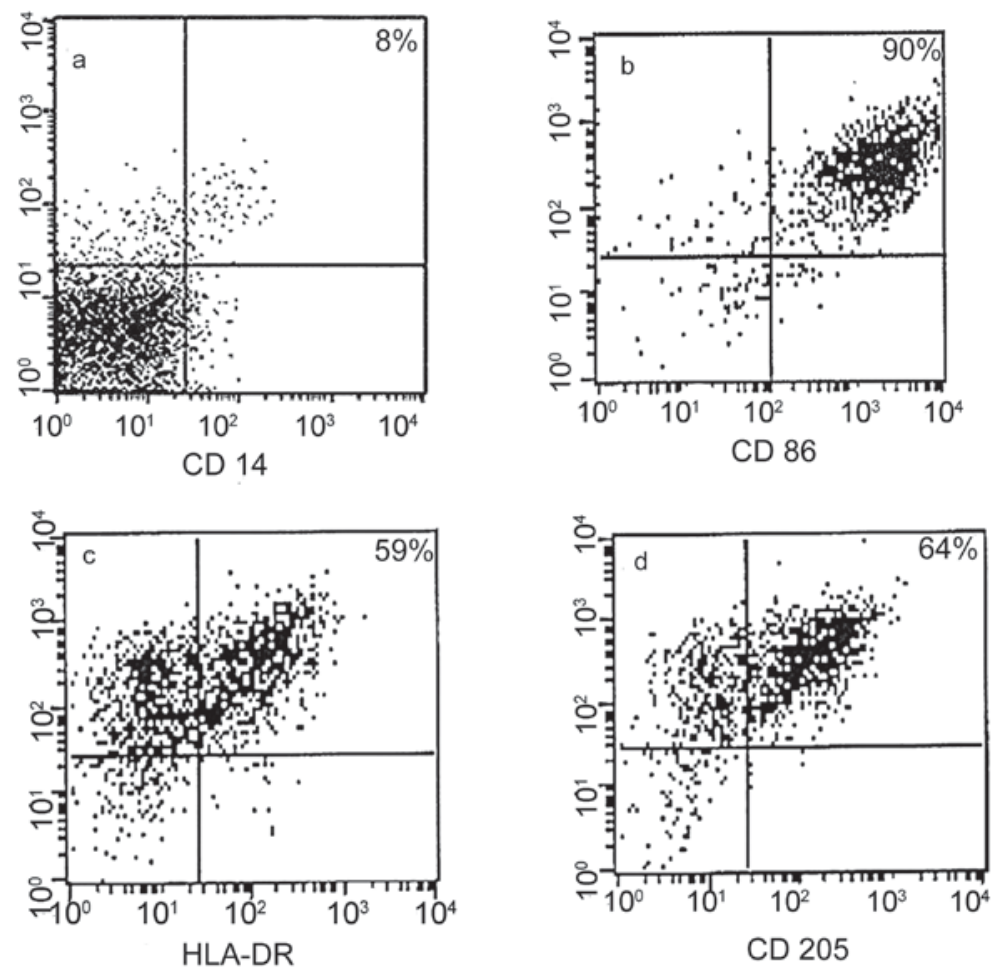

Figure 1. Expression of the CD markers (A) CD14, (B) CD86, (C) HLA-DR and (D) CD205 in the mature dendritic cells. CD, cluster of diffferentiation; HLA-DR, human leukocyte antigen-antigen D related.

interval-scaled variables was tested using Pearson's correlation, whereas the correlation between two ordinal-scaled variables was tested using Spearman's rank correlation. Non-parametric Wilcoxon rank sum test was used to compare the immunological outcomes (e.g. ratio of CD4/CD8 count in blood and IFN- $\gamma$ release from macrophages) between clinical responders $(\mathrm{CD} 4 / \mathrm{CD} 8>1)$ and non-responders (IFN- $\gamma>1 \%$ ). Kaplan-Meier survival analysis was performed using GraphPad Prism software (GraphPad Software, San Diego, CA, USA). 


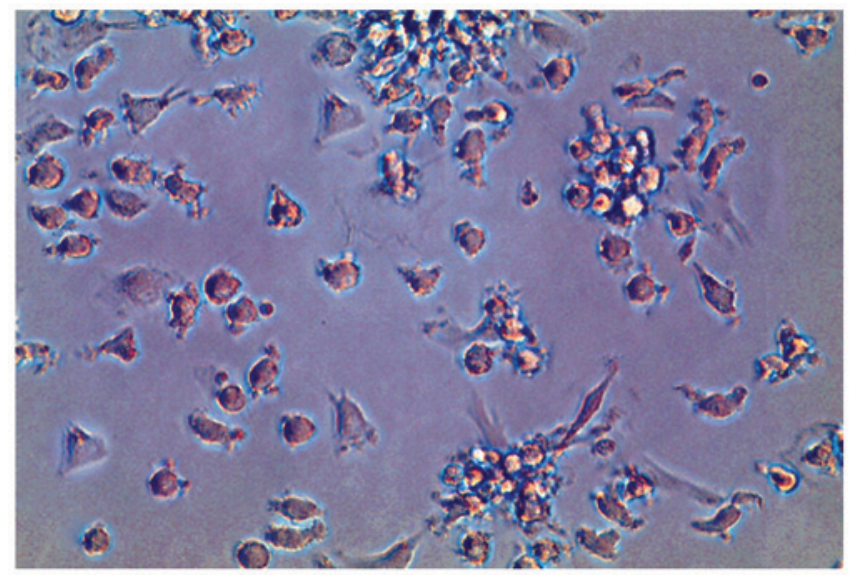

Figure 2. Photomicrograph showing morphology of mature dendritic cells on the day 8 (magnification, $\mathrm{x} 40$ ).

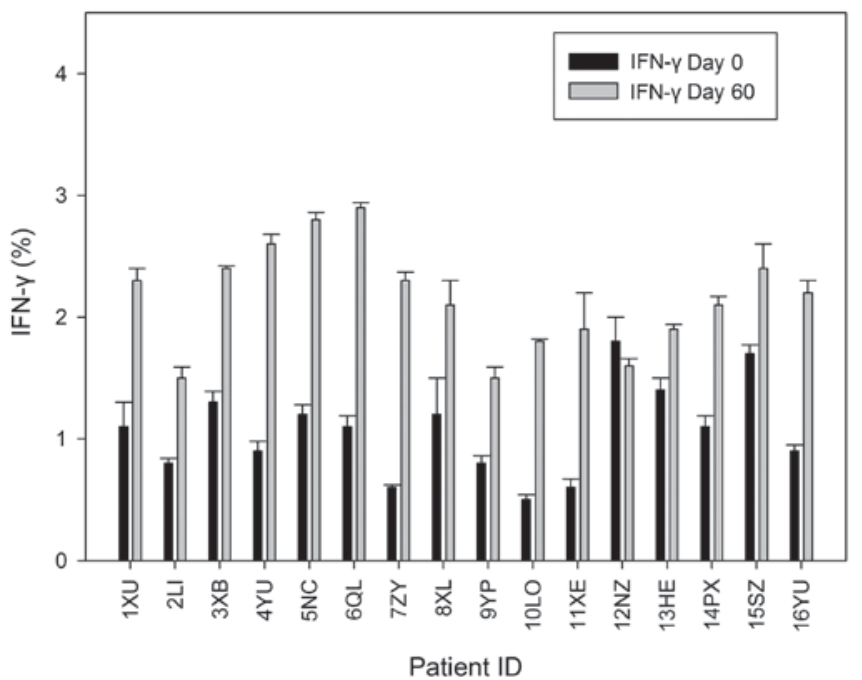

Figure 3. IFN- $\gamma$ levels in individual patients on days 0 and 60 . IFN- $\gamma$, interferon- $\gamma$.

\section{Results}

As aforementioned, 16 patients were enrolled in the present study between December 2013 and October 2014. A total of 19 patients were screened, of which 16 patients met the inclusion criteria. Patient characteristics and clinical outcomes are presented in Table II.

Phenotype of DCs. The final immunotherapy products did not express CD14, and the majority of the cells expressed CD86 (90\%), CD205 (60-75\%) and HLA-DR (55-62\%) (Fig. 1). However, with respect to cytokine secretion, antigen-pulsed $\mathrm{DC} / \mathrm{T}$ cell maturation factor-treated DCs appeared to be more mature compared with naive DCs. Light microscopy of cells cultured for 8 days revealed predominantly mature DCs (Fig. 2).

Delayed-type hypersensitivity test. DCs pulsed with rMAGE3 + rSurvivin were introduced into the forearm intra-dermally to determine DTH reactivity. An induration $>1.5 \mathrm{~cm}$ in diameter was considered as a non-negative DTH

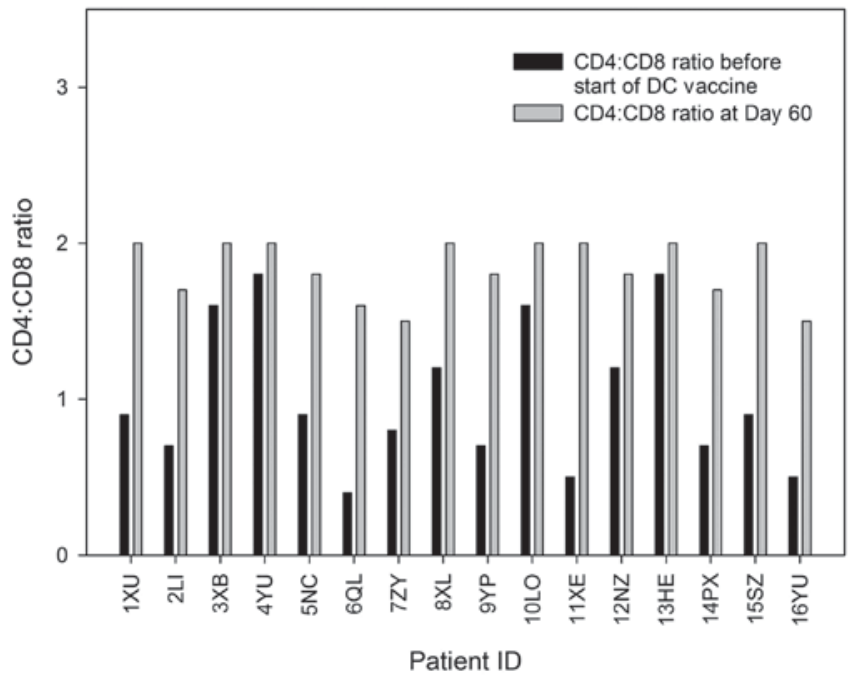

Figure 4. CD4:CD8 ratio evaluated on days 0 and $60 . \mathrm{CD}$, cluster of differentiation.

reaction. Subsequent to the first vaccination, every patient showed a truly positive DTH reaction.

In vitro IFN- $\gamma$ assay. The assay that was elaborated in order to identify the intracellular IFN- $\gamma$ production in the peripheral T cells was utilized to identify the capability of the DC immunotherapy for the progression of an immune response, specifically against tumour cells. The present study used flow cytometry to assess the production of IFN- $\gamma$ in $\mathrm{CD}^{+}$ lymphocytes in patients on days 0 and 60 . In $\mathrm{CD}^{+}$cells that were generated subsequent to T4 (DC immunotherapy arm) and could not be invigorated with ionomycin or PMA, it was observed that there was a significantly increased level of IFN- $\gamma$ expression compared with cells obtained on day $0(\mathrm{P}=0.044)$. IFN- $\gamma$ expression on day 60 was significantly increased compared with day $0(\mathrm{P}=0.48)$ (Fig. 2).

CD4:CD8 levels. There was an increasing trend in the mean CD4:CD8 values between day 30 and day 60 (Fig. 3); however, the increase was not statistically significant $(\mathrm{P}=0.150)$. In the majority of the patients, the basal values were found to be $<2$.

Toxicity. In total, 32 DC injections were subcutaneously administered to the thigh. All injections were well tolerated; however, one incident of temporary exanthema was observed in one patient (patient ID, 5NC). It was observed that the exanthema vanished without any supplementary treatment. At the DC injection region, $18.75 \%$ (3/16) of patients reported a small itching induration. No patients showed any serious adverse events. Overall, DC immunotherapy was found to be safe and well tolerated and only incidence of grade 1 chills, fever and fatigue was observed.

Response evaluation. At least 12 months of follow up was performed for all patients subsequent to primary immunisation, and the clinical follow-up data are shown in Table II. The disease recurred or progressed in 5 patients, 3 of which succumbed to NSCLC 4-9 months after detection due to disease progression to an advanced stage (stage IV). One 
NSCLC patient (patient ID, DC10), who had stage I disease and had received radiotherapy and chemotherapy, developed solitary brain metastasis 2 months subsequent to T0. Subsequently, 15 months after local resection of stage IV disease, the patient showed no evidence of NSCLC. One patient with stage IIIB unresectable disease developed local progression 16 months subsequent to T0 and 19 months subsequent to chemoradiation. A sixth patient (patient ID, DC16) with stage IIIA disease that could be resected surgically and had received multimodality therapy developed radiographically persistent nodule 12 months subsequent to T0 and 21 months subsequent to completion of treatment and was currently receiving chemotherapy.

\section{Discussion}

The current study has validated the concept of cellular immunotherapy using MODCs as a viable treatment option in treating NSCLC. No patients demonstrated treatment-associated haematological, hepatic, renal or neurological toxicity, or autoimmune disease, indicating that the autologous DC immunotherapy was safe. DCs also met the specifications for quality control described by Sabado et al (18). Aggressive treatment of NSCLC has lead to improved outcomes $(19,20)$, and survival can further be increased by expanding the scope of available therapeutic options for NSCLC $(21,22)$. Immunotherapy specifically targets malignant cells and is an attractive systemic approach. Evidence that autologous tumour immunotherapy expressing GM-CSF (GVAX) elicits a durable clinical response in patients with NSCLC indicates that it is possible to modulate the immune system to benefit NSCLC patients (14). Although it is questionable whether immunotherapy can adequately and consistently treat such considerable diseases, efficient immunotherapy can act as an adjuvant therapy for surgical multimodality or medical therapy that shows definitive clinical responses. The ultimate objective of the present study was to identify the role of immunotherapy as an adjuvant therapy in the treatment of stage I-IIIB NSCLC. Therefore, the main aim of the current study was to identify the immunological response generated by autologous DC immunotherapy in 16 patients with NSCLC. In total, 5 patients experienced disease recurrence or progression, of which 3 patients succumbed to disease progression. In addition, 3 patients experienced therapeutic efficacy. One patient, who had stage IB disease, developed solitary brain metastasis 2 months subsequent to vaccination (DC immunotherapy); however, following surgical resection of stage IV disease, the patient survived for 15 months. Additionally, no disease progression was observed in 2 patients with stage III unresectable disease 23 months and 35 months after chemoradiation, respectively. One patient with bronchoalveolar carcinoma, who had resected stage IIIB disease, also remained tumour-free 19 months subsequent to vaccination and 28 months subsequent to surgical resection.

A positive DTH response against the TAAs used for priming the DC was observed in all patients. Previous studies have not shown a statistically significant increase in the release of IFN- $\gamma(17,18)$. However, the levels of IFN- $\gamma$ released by $\mathrm{CD}^{+}$ cells in the present study support the activation of the immune response by DC immunotherapy. In the present study, MAGE3 and Survivin were used as TAAs for DC immunotherapy and for generating a Th1 immune response; the use of purified and defined MAGE3 and Survivin peptides to prime DCs has already been demonstrated $(23,24)$.

Therapeutic cancer immunotherapies have become a reality (17). Initial failures have increased knowledge of the immune response against tumours and prompted the development of immunotherapies and immunotherapeutic agents that are more potent and considerably less toxic than chemotherapies or targeted therapies $(6,10)$. Trials and approval of the first DC immunotherapy in the US have shown that activating the immune system with a therapeutic cancer immunotherapy can provide clinical benefit to cancer patients for a prolonged period (25). Immunotherapies have been more successful in prostate cancer due to the generally indolent progression of prostate cancer (25). In the present study, only patients with advanced tumour stage were enrolled; however, the optimal setting to apply DC immunotherapy may be minimal residual disease. The foci of on-going and forthcoming studies are various aspects of immunotherapy optimisation, antigen preparation and methods of application (26). The current study showed that DCs can be used in adoptive immunotherapy for the treatment of NSCLC. However, if these promising results can be confirmed in a larger patient population, then DC immunotherapy based on the combination of rMAGE3 and rSurvivin may become a sought after option for treating NSCLC. Several questions associated with the manufacturing and quality of immunotherapy, immune monitoring, patient selection and immunotherapy delivery strategies need to be addressed. Future studies should address prominent issues, including secretion of immunosuppressive cytokines and mechanisms of tumour escape from immune surveillance, through down-regulation of antigen and MHC expression.

\section{References}

1. Molina JR, Yang P, Cassivi SD, Schild SE and Adjei AA: Non-small cell lung cancer: Epidemiology, risk factors, treatment, and survivorship. Mayo Clinic Proc 83: 584-594, 2008.

2. Reck M: What future opportunities may immuno-oncology provide for improving the treatment of patients with lung cancer? Ann Oncol 23 (suppl 8): viii28-viii34, 2012.

3. Romani N, Reider D, Heuer M, Ebner S, Kämpgen E, Eibl B, Niederwieser D and Schuler G: Generation of mature dendritic cells from human blood. An improved method with special regard to clinical applicability. J Immunol Methods 196: 137-151, 1996.

4. Sallusto F and Lanzavecchia A: Efficient presentation of soluble antigen by cultured human dendritic cells is maintained by granulocyte/macrophage colony-stimulating factor plus interleukin 4 and downregulated by tumor necrosis factor alpha. J Exp Med 179: 1109-1118, 1994

5. He L, Feng H, Raymond A, Kreeger M, Zeng Y, Graner M, Whitesell L and Katsanis E: Dendritic-cell-peptide immunization provides immunoprotection against bcr-abl-positive leukemia in mice. Cancer Immunol Immunother 50: 31-40, 2001.

6. Mayordomo JI, Zorina T, Storkus WJ, Zitvogel L, Celluzzi C, Falo LD, Melief CJ, Ildstad ST, Kast WM, Deleo AB, et al: Bone marrow-derived dendritic cells pulsed with synthetic tumour peptides elicit protective and therapeutic antitumour immunity. Nat Med 1: 1297-1302, 1995.

7. Schuler G and Steinman RM: Dendritic cells as adjuvants for immune-mediated resistance to tumors. J Exp Med 186: 1183-1187, 1997.

8. Banchereau J and Steinman RM: Dendritic cells and the control of immunity. Nature 392: 245-252, 1998.

9. Fong L, Hou Y, Rivas A, Benike C, Yuen A, Fisher GA, Davis MM and Engleman EG: Altered peptide ligand vaccination with Flt3 ligand expanded dendritic cells for tumor immunotherapy. Proc Natl Acad Sci USA 98: 8809-8814, 2001. 
10. Nair SK, Hull S, Coleman D, Gilboa E, Lyerly HK and Morse MA Induction of carcinoembryonic antigen (CEA)-specific cytotoxic T-lymphocyte responses in vitro using autologous dendritic cells loaded with CEA peptide or CEA RNA in patients with metastatic malignancies expressing CEA. Int J Cancer 82: 121-124, 1999.

11. Falleni M, Pellegrini C, Marchetti A, Oprandi B, Buttitta F, Barassi F, Santambrogio L, Coggi G and Bosari S: Survivin gene expression in early-stage non-small cell lung cancer. J Pathol 200: 620-626, 2003.

12. Weiser TS, Ohnmacht GA, Guo ZS, Fischette MR, Chen GA, Hong JA, Nguyen DM and Schrump DS: Induction of MAGE-3 expression in lung and esophageal cancer cells. Ann Thorac Surg 71: 295-302, 2001.

13. US Department of Health and Human Services, National Institutes of Health, National Cancer Institute: Common terminology criteria for adverse events, version, 4.0. https://evs.nci.nih.gov/ftpl/ CTCAE/CTCAE_4.03_2010-06-14_QuickReference_5x7.pdf. Accessed October, $201 \overline{4}$.

14. Hirschowitz EA, Foody T, Kryscio R, Dickson L, Sturgill J and Yannelli J: Autologous dendritic cell vaccines for non-small-cell lung cancer. J Clin Oncol 22: 2808-2815, 2004.

15. Kim D, Jeon C, Kim JH, Kim MS, Yoon CH, Choi IS, Kim SH and Bae YS: Cytoplasmic transduction peptide (CTP): New approach for the delivery of biomolecules into cytoplasm in vitro and in vivo. Exp Cell Res 312: 1277-1288, 2006.

16. Kern F, Surel IP, Brock C, Freistedt B, Radtke H, Scheffold A, Blasczyk R, Reinke P, Schneider-Mergener J, Radbruch A, et al: T-cell epitope mapping by flow cytometry. Nat Med 4: 975-978, 1998.

17. Bapsy PP, Sharan B, Kumar C, Das RP, Rangarajan B, Jain M, Suresh Attili VS, Subramanian S, Aggarwal S, Srivastava M and Vaid A: Open-label, multi-center, non-randomized, single-arm study to evaluate the safety and efficacy of dendritic cell immunotherapy in patients with refractory solid malignancies, on supportive care. Cytotherapy 16: 234-244, 2014.
18. Sabado RL, Miller E, Spadaccia M, Vengco I, Hasan F and Bhardwaj N: Preparation of tumor antigen-loaded mature dendritic cells for immunotherapy. J Vis Exp, 2013.

19. Spira A and Ettinger DS: Multidisciplinary management of lung cancer. N Engl J Med 350: 379-392, 2004

20. Arriagada R, Bergman B, Dunant A, Le Chevalier T, Pignon JP and Vansteenkiste J; International Adjuvant Lung Cancer Trial Collaborative Group: Cisplatin-based adjuvant chemotherapy in patients with completely resected non-small-cell lung cancer. $\mathrm{N}$ Engl J Med 350: 351-360, 2004.

21. Dy GK and Adjei AA: Novel targets for lung cancer therapy: Part II. J Clin Oncol 20: 3016-3028, 2002.

22. Dy GK and Adjei AA: Novel targets for lung cancer therapy: Part I. J Clin Oncol 20: 2881-2894, 2002.

23. Liu X, Sun N, Dong Y, Li J, Liu Y, Ren Y, Yang C, Zhang L, Zhou Y, Tong Z, et al: Anticancer effects of adenovirus-mediated calreticulin and melanoma-associated antigen 3 expression on non-small cell lung cancer cells. Int Immunopharmacol 25: 416-424, 2015.

24. Hobo W, Strobbe L, Maas F, Fredrix H, Greupink-Draaisma A, Esendam B, de Witte T, Preijers F, Levenga H, van Rees B, et al: Immunogenicity of dendritic cells pulsed with MAGE3, Survivin and B-cell maturation antigen mRNA for vaccination of multiple myeloma patients. Cancer Immunol Immunother 62: 1381-1392, 2013.

25. Cheever MA and Higano CS: PROVENGE (Sipuleucel-T) in prostate cancer: The first FDA-approved therapeutic cancer vaccine. Clin Cancer Res 17: 3520-3526, 2011.

26. Tuyaerts S, Aerts JL, Corthals J, Neyns B, Heirman C, Breckpot K, Thielemans K and Bonehill A: Current approaches in dendritic cell generation and future implications for cancer immunotherapy. Cancer Immunol Immunother 56: 1513-1537, 2007. 